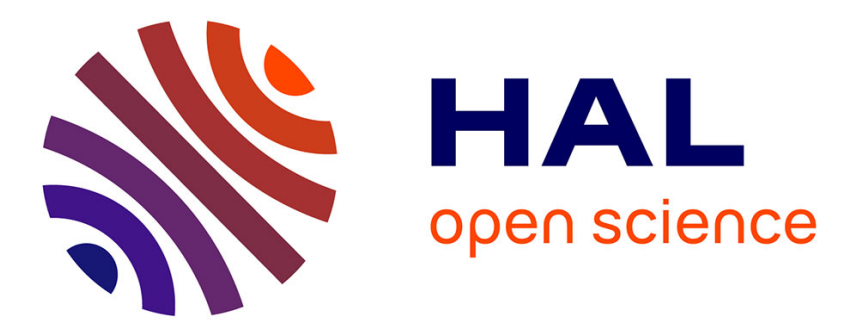

\title{
Variability of T1-weighted signal intensity of pericallosal lipomas in the fetus
}

\author{
Lydia Chougar, Eléonore Blondiaux, Marie-Laure Moutard, Antoinette Gelot, \\ Jean-Marie Jouannic, Hubert Ducou Le Pointe, Catherine Garel
}

\section{- To cite this version:}

Lydia Chougar, Eléonore Blondiaux, Marie-Laure Moutard, Antoinette Gelot, Jean-Marie Jouannic, et al.. Variability of T1-weighted signal intensity of pericallosal lipomas in the fetus. Pediatric Radiology, In press, pp.1-9. 10.1007/s00247-017-4028-1 . hal-01675273

\section{HAL Id: hal-01675273 \\ https://hal.sorbonne-universite.fr/hal-01675273}

Submitted on 4 Jan 2018

HAL is a multi-disciplinary open access archive for the deposit and dissemination of scientific research documents, whether they are published or not. The documents may come from teaching and research institutions in France or abroad, or from public or private research centers.
L'archive ouverte pluridisciplinaire HAL, est destinée au dépôt et à la diffusion de documents scientifiques de niveau recherche, publiés ou non, émanant des établissements d'enseignement et de recherche français ou étrangers, des laboratoires publics ou privés. 


\title{
Variability of T1-weighted signal intensity of pericallosal lipomas in the fetus
}

\author{
Lydia Chougar $^{1}$ - Eléonore Blondiaux ${ }^{1}$ - Marie-Laure Moutard ${ }^{2} \cdot$ Antoinette Gelot $^{3}$. \\ Jean-Marie Jouannic ${ }^{4} \cdot$ Hubert Ducou Le Pointe $^{1} \cdot$ Catherine Garel $^{1}$
}

\begin{abstract}
Background Pericallosal lipomas are often associated with corpus callosum dysgenesis. The diagnosis of lipoma, suggested on ultrasonography, relies on the classic T1 hyperintensity on magnetic resonance imaging (MRI). However, this feature may be absent prenatally.

Objective Our objective was to study the changes of $\mathrm{T} 1$ intensity in fetal lipomas with comparison to postnatal/ postmortem data and to assess the factors influencing the signal variations of pericallosal lipomas on prenatal MRI.

Materials and methods Patients with callosum dysgenesis and interhemispheric hyperechogenicity suggestive of a pericallosal lipoma with available postnatal or postmortem data were included. Gestational age, lipoma size and pattern, corpus callosum size and changes in fetal fat $\mathrm{T} 1$ intensity were recorded. Comparison with postmortem neuropathology was available for one fetus.
\end{abstract}

Catherine Garel

catherine.garel@aphp.fr

1 Service de Radiologie,

Hôpital Trousseau - Hôpitaux Universitaires

de l'Est Parisien (APHP),

Université Pierre et Marie Curie, Paris, France

2 Service de Neurologie Pédiatrique, APHP,

Hôpitaux Universitaires de l'Est Parisien,

Hôpital Trousseau, Paris, France

3 Service d'Anatomie Pathologique,

Hôpital Trousseau - Hôpitaux Universitaires

de l'Est Parisien (APHP),

Université Pierre et Marie Curie, Paris, France

4 Service de Médecine Fœtale,

Hôpital Trousseau - Hôpitaux Universitaires

de l'Est Parisien (APHP),

Sorbonne Universités, UPMC Univ Paris 06, Paris, France
Results Eleven patients with callosum dysgenesis and pericallosal lipomas (seven curvilinear and four tubulonodular) were included. All MRI scans were performed in the third trimester. Curvilinear lipomas were thinner and six cases were associated with prenatal T1 iso-intensity. Typical T1 hyperintensity appeared on postnatal MRI only. All tubulonodular lipomas were much larger and showed prenatal T1 hyperintensity. In two patients, the lipoma increased in size on postnatal MRI.

Conclusion The type and size of a lipoma influence T1 prenatal intensity. Absence of T1 intensity was observed in curvilinear lipomas only. Curvilinear lipomas are much thinner. Changes in $\mathrm{T} 1$ intensity may also be related to fat maturation within the lipoma and, subsequently, to gestational age. In the case of callosum dysgenesis, absence of prenatal T1 pericallosal hyperintensity should not exclude the diagnosis of pericallosal lipoma.

Keywords Brown adipose tissue $\cdot$ Corpus callosum dysgenesis $\cdot$ Fetus $\cdot$ Magnetic resonance imaging $\cdot$ Pericallosal lipoma $\cdot$ Ultrasonography $\cdot$ White adipose tissue

\section{Introduction}

Intracranial lipomas are rare congenital malformations [1-4]. Pericallosal lipomas represent the most common location and are often associated with corpus callosum dysgenesis, as both anomalies occur at the same stage of embryogenesis [1-7]. Corpus callosum dysgenesis carries a better prognosis if a pericallosal lipoma is present $[7,8]$.

Postnatally, pericallosal lipomas display typical T1 hyperintensity on magnetic resonance imaging (MRI) [1-5]. Prenatally, lipomas are also reported as being T1 hyperintense [6-8]. 
Our objective was to study the variability of signal intensity in pericallosal lipomas on T1-weighted sequences with correlation to postnatal/postmortem data and to assess the factors influencing the signal variations of pericallosal lipomas on prenatal MRI.

\section{Materials and methods}

\section{Inclusion criteria}

This was a retrospective study conducted in an institution including a tertiary prenatal center and a pediatric hospital. Pregnant women may be referred to the department of pediatric radiology following detection of a short corpus callosum. For this study, prenatal ultrasonography (US) had been performed by a pediatric radiologist (C.G.) with 25 years of experience in prenatal ultrasound. The MRI images were read by two pediatric radiologists with 7 and 28 years of experience in prenatal MRI, respectively (E.B., C.G.). We retrieved from our database the cases of proven pericallosal lipoma with prenatal imaging including US and MRI from September 2006 to November 2016. Informed consent was obtained from the patients and the study was conducted in accordance with the institutional review board.

The inclusion criteria were the following: availability of a second-line prenatal US suspecting pericallosal lipoma on the basis of an interhemispheric hyperechogenicity similar to bone echogenicity, association with a corpus callosum dysgenesis (i.e. incomplete, short and sometimes too thick corpus callosum) [7-9], availability of prenatal MRI images, and confirmation of pericallosal lipoma either by neuropathological examination in case of termination of pregnancy or by postnatal MRI displaying typical disappearing T1 interhemispheric hyperintensity on fat-suppressed sequences.

\section{Prenatal and postnatal imaging parameters}

US was performed using an Aplio (Toshiba, Tokyo, Japan) system with transabdominal probes (3.5 to $14 \mathrm{MHz}$ low and high frequency probes) or transvaginal probes $(6 \mathrm{MHz})$ when required by the fetal presentation. MRI scans were performed using a 1.5-T unit (Philips Medical System, Achieva model; Best, the Netherlands, in 8 cases; Optima; General Electric, Milwaukee, WI, in 3 cases). Prenatal MRI protocol included at least T1-weighted fast spin echo sequences (TR $382 \mathrm{~ms} / \mathrm{TE}$ $14 \mathrm{~ms}$ ), T2-weighted ultra-fast spin echo single shot (TR $15,000 \mathrm{~ms} / 120 \mathrm{~ms}$ ), and T1-weighted spin echo with fat suppression sequences (TR $693 \mathrm{~ms} / \mathrm{TE} 14 \mathrm{~ms}$ ) in the sagittal and coronal or axial planes and thickness: $4 \mathrm{~mm}$ with a $1 \mathrm{~mm}$-gap.

Postnatal MRI protocol included at least T1-weighted spin echo sequences (TR $561 \mathrm{~ms} / \mathrm{TE} 14 \mathrm{~ms}$ ) in the sagittal plane, T2-weighted fast spin echo sequences in the sagittal (TR/TE
$3,500 \mathrm{~ms} / 120 \mathrm{~ms}$ ) or coronal (TR/TE $6,071 \mathrm{~ms} / 100 \mathrm{~ms}$ ) plane, and T1-weighted sequences with fat suppression (TR/TE $539 \mathrm{~ms} / 14 \mathrm{~ms}$ ) in the sagittal plane. Slice thickness was $2 \mathrm{~mm}$ to $4 \mathrm{~mm}$ with a $0.2 \mathrm{~mm}$-gap.

\section{Pericallosal lipomas analysis}

In each case, the following data were recorded: The type of lipoma, curvilinear or tubulonodular, was defined as follows. The tubulonodular type was characterized by a bulky mass (superior to $2 \mathrm{~cm}$ ) that could be anterior or posterior. The curvilinear type was thin and long, measuring less than $1 \mathrm{~cm}$ in diameter, often posterior and surrounded the superior aspect of the corpus callosum [1-11].

- Size of the lipoma on prenatal and postnatal images for tubulonodular lipomas and on postnatal images for curvilinear lipomas. Measurements included anteroposterior diameter, height and lateral diameter for tubulonodular lipomas and anteroposterior and lateral diameters only for curvilinear lipomas. The height was always inferior to $3 \mathrm{~mm}$ and its measurement was considered inaccurate.

- Corpus callosum appearance and anteroposterior diameter (measured between the rostrum and the splenium), on prenatal US images and on pre- and postnatal MRI scans: This diameter was evaluated according to published reference data for prenatal US [12] and pre- and postnatal MRI $[13,14]$.

- Gestational age at first US scan considered pathological, at prenatal MRI and age at postnatal MRI.

- Lipoma T1 intensity at pre-and postnatal MRI.

- Subcutaneous fat tissue intensity at prenatal MRI.

Autopsy with neuropathological analysis was performed in one fetus (patient 1 in Table 1) and included immunohistochemical evaluation (using S-100, which is an immunochemical marker of adipocytes [15]).

The children underwent neurological examination at birth and during follow-up.

\section{Results}

The inclusion criteria were fulfilled in 11 fetuses. In one fetus with a tubulonodular lipoma (patient 1), the pregnancy was terminated in accordance with French legislation, due to the complete absence of corpus callosum associated with a large tubulonodular lipoma and subsequent risk for mental deficiency and seizures. The results are summarized in Table 1.

A curvilinear lipoma was found in seven fetuses and a tubulonodular type in four fetuses (three were anterior and one was posterior). A complete corpus callosum agenesis was observed in three fetuses and was associated with a 


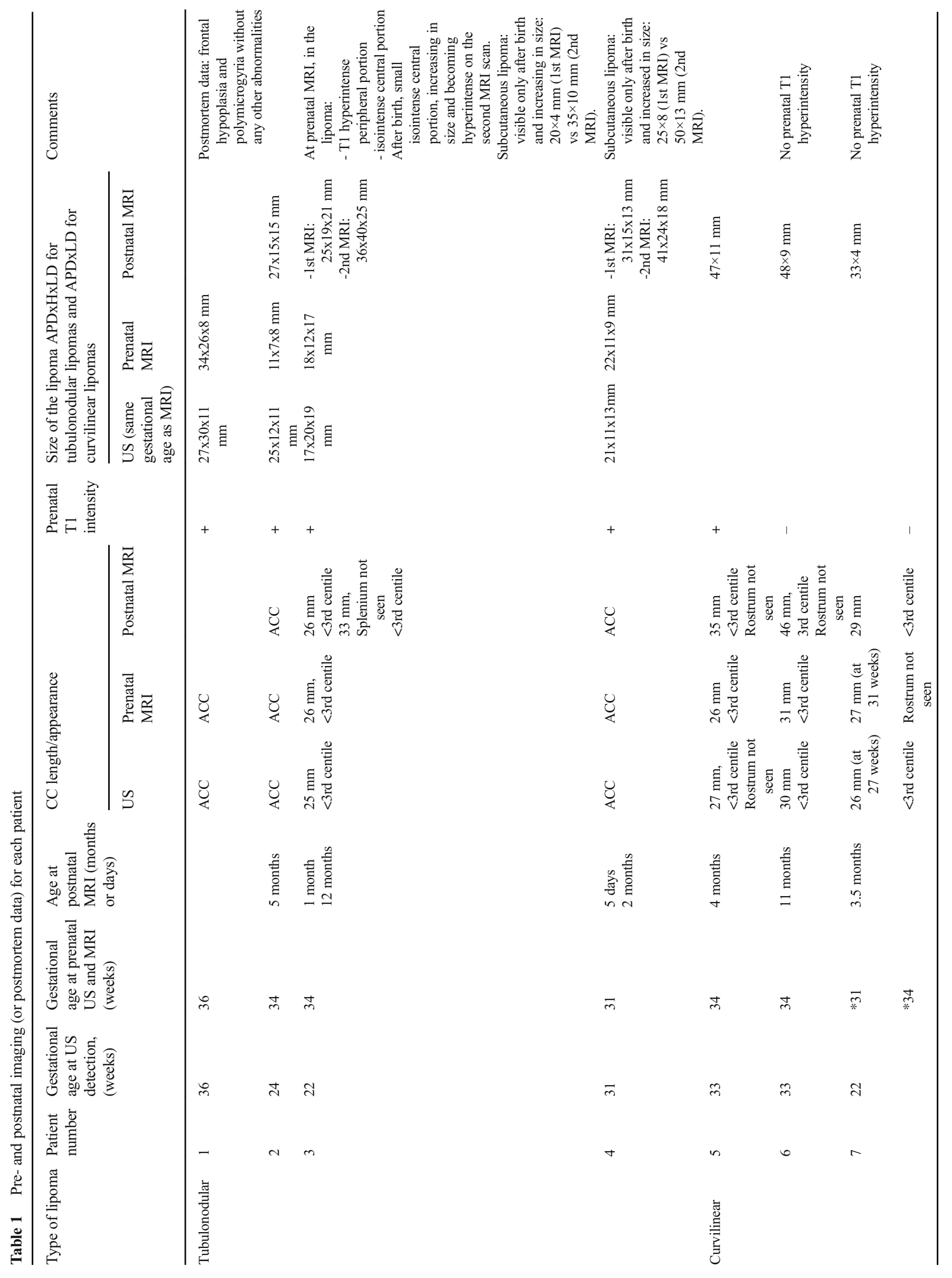




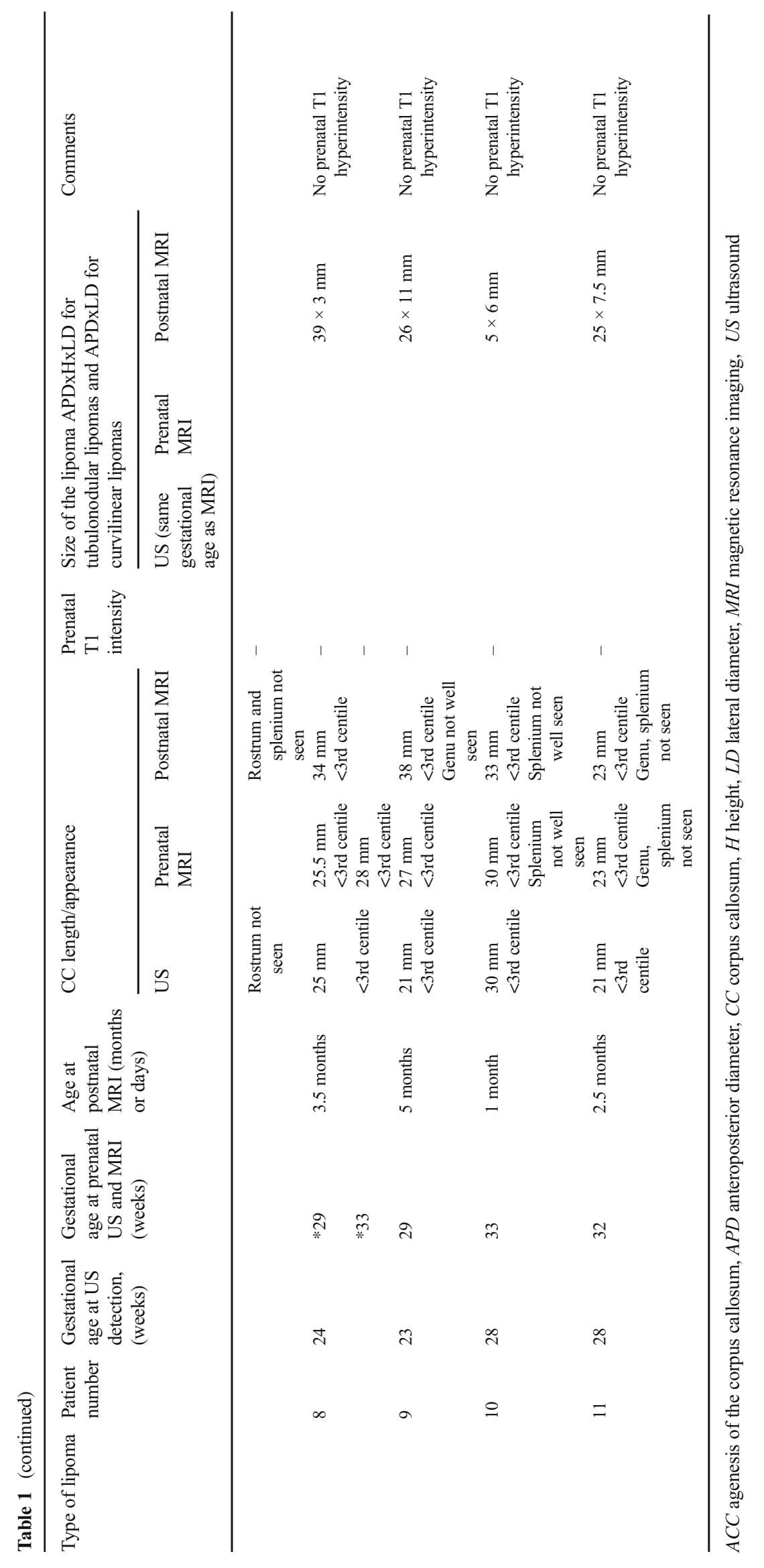


tubulonodular lipoma. In the other eight fetuses, a short corpus callosum (with an anteroposterior diameter inferior to the third centile) was present.

The diagnosis of lipoma was suggested at US in the second trimester (between 22 and 28 weeks of gestation) in 7 fetuses and in the third trimester (between 31 and 36 weeks of gestation) in 4 fetuses. The postnatal MRI scans were performed between birth and 1 year of age.

In all cases of tubulonodular lipomas (patients 1 to 4 ) at prenatal MRI, T1 hyperintensity was present and disappeared after fat suppression. The lateral diameter varied between 11 and $19 \mathrm{~mm}$ at prenatal US and 8 and $17 \mathrm{~mm}$ at prenatal MRI. In two fetuses (patients 3 and 4), an increase in size of the tubulonodular lipoma between pre- and postnatal images, on one hand, and between the two postnatal MRI scans, on the other hand, was observed. A subcutaneous lipoma was associated in these two cases, also displaying an increase in size (Fig. 1). In another fetus with a tubulonodular lipoma (patient 2), the size of the lipoma was obviously underestimated on the prenatal MRI in comparison with the US findings. After birth, the lipoma appeared larger and more extensive, similar to the prenatal sonographic appearance.

In the 7 fetuses with a curvilinear lipoma (patients 5 to 11) at prenatal MRI, T1 hyperintensity was present in only one case (patient 5) with MRI being performed at 34 weeks of gestation (Fig. 2). In the six remaining fetuses, T1 hyperintensity was absent. MRI was performed at 34 weeks of gestation (patient 6), 31-34 weeks of gestation (patient 7), 29-33 weeks of gestation (patient 8), 29 weeks of gestation (patient 9), 33 weeks of gestation (patient 10) and 32 weeks of gestation (patient 11), respectively. In these 6 patients, T1 hyperintensity was observed after birth, between 2.5 and 11 months of age (Fig. 3). In curvilinear lipomas, hyperechogenicity was poorly delineated and, therefore, measurements were inaccurate. Because most curvilinear lipomas are overlooked prenatally, measurements were acquired on postnatal MR images. The lateral diameter varied between $3 \mathrm{~mm}$ and $11 \mathrm{~mm}$. The only curvilinear lipoma being identified on prenatal MRI (patient 5) showed the largest lateral diameter and was globally rather thick. The other lipoma with such a large lateral diameter (patient 9) was thick posteriorly only and otherwise thin $(<3 \mathrm{~mm})$.

In all cases of pericallosal lipoma, the subcutaneous fat layer displayed T1 hyperintensity. In patients 8 and 9, MRI was performed at 29 weeks of gestation and $\mathrm{T} 1$ hyperintensity was less prominent compared with examinations performed later in this series, including the second MRI performed at 33 weeks in case 8 .

In patient 1 , the macroscopic analysis revealed pathological dura mater filled with adipose tissue within the interhemispheric fissure. The corpus callosum was absent and the frontal lobes were hypoplastic. The underlying cortex was polymicrogyric. At histological analysis, the lipoma contained two types of cells, brown and white adipocytes, the latter being predominant, mostly in a juxtacortical location (Fig. 4).

The ten surviving patients had normal neurological examinations at birth. The follow-ups lasted between 1 month and 5 years. One patient with a tubulonodular lipoma (patient 4) developed a tonicoclonic form of epilepsy associated with a
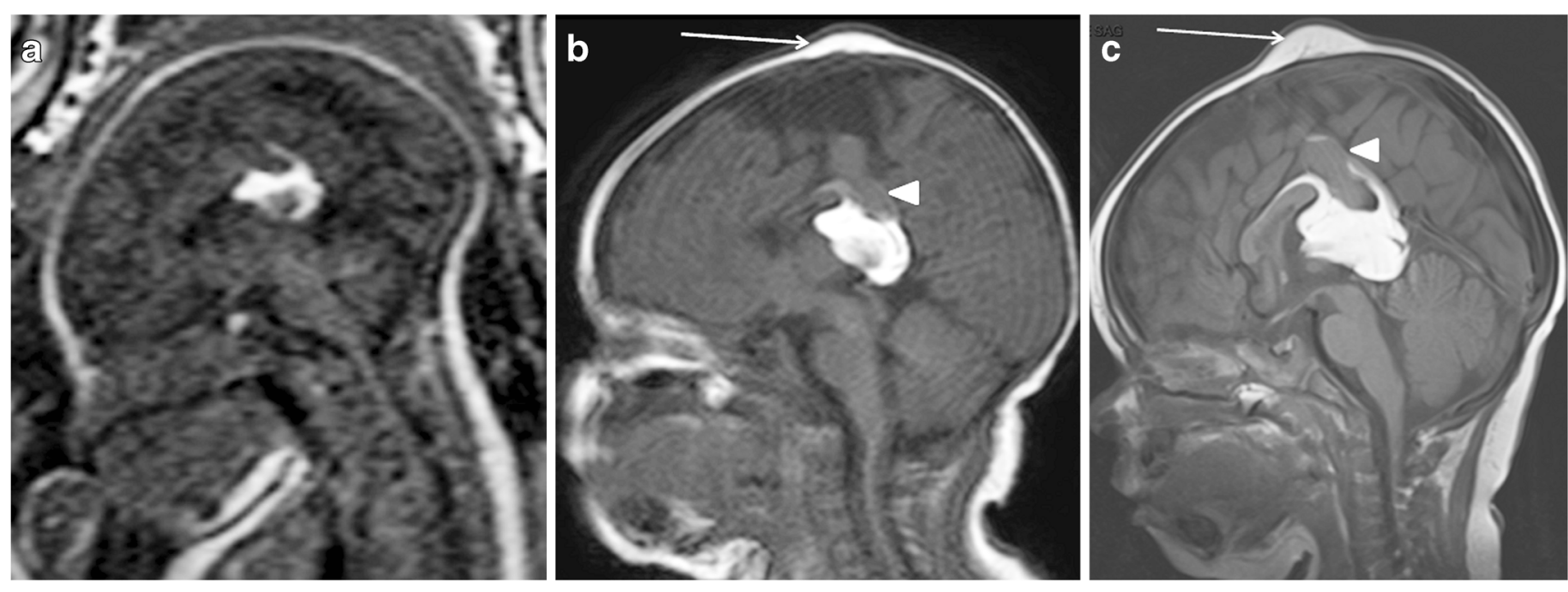

Fig. 1 Tubulonodular lipoma (patient 3 in Table 1). a Prenatal brain MRI performed at 34 weeks' gestation, midline T1-weighted sagittal slice without fat suppression $(\mathrm{TR}=382 \mathrm{~ms}, \mathrm{TE}=14 \mathrm{~ms})$. The peripheric portion of the lipoma is $\mathrm{T} 1$ hyperintense while the central portion is isointense to the cerebral parenchyma. b, $\mathbf{c}$ Postnatal sagittal brain MRI performed at 1 month $(\mathbf{b})(\mathrm{TR}=500 \mathrm{~ms}, \mathrm{TE}=12 \mathrm{~ms})$ and 12 months of age (c) $(\mathrm{TR}=600 \mathrm{~ms}, \mathrm{TE}=9 \mathrm{~ms})$, respectively, midline $\mathrm{T} 1$-weighted sagittal slice without fat suppression. The central portion of the lipoma is isointense at the age of 1 month and is markedly hyperintense at the age of 12 months. The pericallosal lipoma has increased in size after birth as well as the frontal subcutaneous lipoma (arrow in b,c), which was not visible before birth. Some signal voids are visible within both pericallosal and subcutaneous lipomas, in relation to vessels. Above the pericallosal lipoma, a mass (arrowhead), possibly a hamartoma, shows iso-T1 and T2 signal intensity, similar to brain signal intensity 

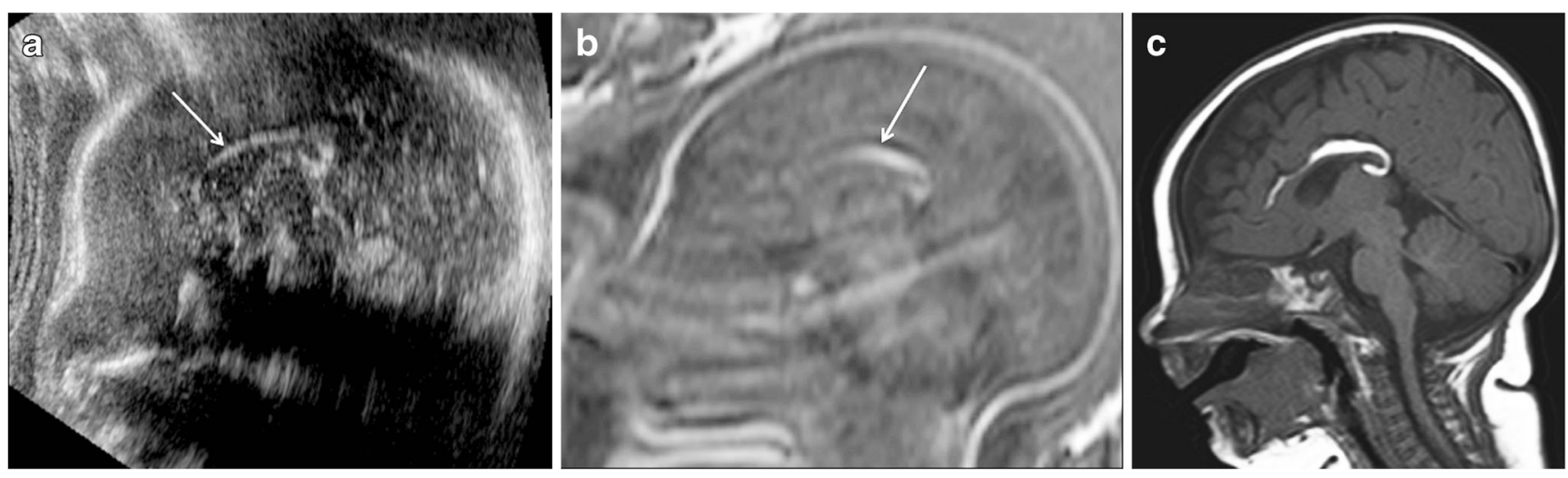

Fig. 2 Curvilinear lipoma with prenatal T1 hyperintensity (case 5 in Table 1). a Prenatal brain US performed at 33 weeks' gestation, midline sagittal view, shows a linear hyperechogenicity (arrow) above the corpus callosum. b Prenatal brain MRI performed at 34 weeks' gestation is midline $\mathrm{T} 1$-weighted sagittal slice without fat suppression $(\mathrm{TR}=382 \mathrm{~ms}$,

mild psychomotor retardation. The remaining patients had normal development.

\section{Discussion}

The presence of $\mathrm{T} 2$ signal hypointensity and $\mathrm{T} 1$ signal hyperintensity disappearing on fat-suppressed sequences is a strong argument in favor of a pericallosal lipoma; T2 signal hypointensity may be quite subtle while T1 signal hyperintensity, when present, is much more conspicuous. This is why this study focused on T1 signal intensity. It shows that $\mathrm{T} 1$ hyperintensity of pericallosal lipomas may be absent on prenatal MRI. This finding was observed in most cases of curvilinear lipomas. Conversely, T1 hyperintensity was
$\mathrm{TE}=14 \mathrm{~ms}$ ). The quality of the image is poor due to fetal movements. However, the lipoma (arrow) is readily visible and appears T1 hyperintense. c Postnatal brain MRI performed at 4 months is midline T1-weighted sagittal slice without fat suppression $(\mathrm{TR}=561 \mathrm{~ms}$, $\mathrm{TE}=14 \mathrm{~ms}$ ). The hyperintence curvilinear lipoma is well visible

always present in tubulonodular lipomas, but in some cases, additional areas of $\mathrm{T} 1$ hyperintensity within the lipoma were visible after birth only. Two factors accounting for these results could be identified: The first factor is the size of the lipoma, curvilinear lipomas being much thinner than tubulonodular ones, and the second factor might be histological maturation of lipomas and changes in the adipose tissue properties.

According to the literature, intracranial lipomas are considered congenital malformations [1-4] resulting from abnormal differentiation of the primitive meninx or benign mesenchymal tumors $[5,6]$. Pericallosal lipomas are rare lesions, with all intracranial lipomas accounting for only $0.1 \%$ to $1 \%$ of all intracranial tumors $[2,3]$. Two patterns are described: the tubulonodular type characterized by a bulky mass that can
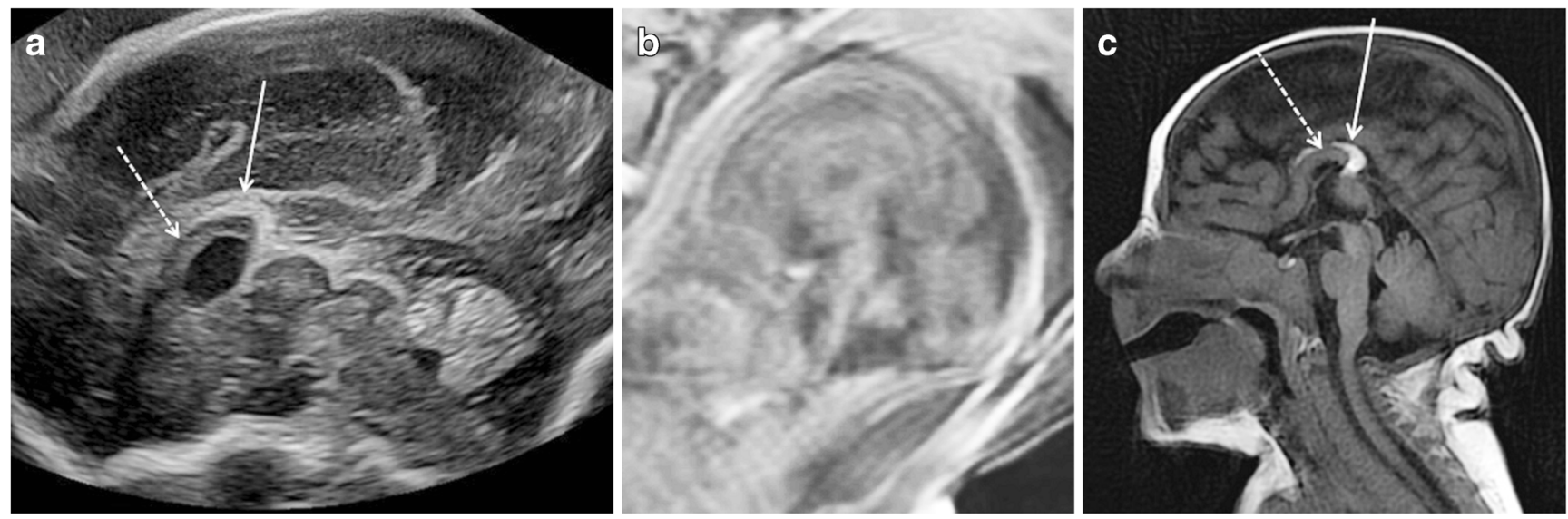

Fig. 3 Curvilinear lipoma without prenatal T1 hyperintensity (case 11 in Table 1). a Prenatal brain US scan performed at 28 weeks' gestation, midline sagittal view, shows a posterior linear hyperechoic lesion (arrow) located above and behind the posterior part of the corpus callosum (dashed arrow). The corpus callosum is short. b Prenatal brain MRI performed at 32 weeks' gestation is midline T1-weighted sagittal slice without fat suppression $(\mathrm{TR}=160 \mathrm{~ms}$, TE=4 ms). No midline T1 hyperintensity is visible. c Postnatal MRI at 2.5 months of age is midline T1 IR-weighted slice (TR=2,997 ms, TE=48 ms). The curvilinear lipoma (arrow) is well visible. The corpus callosum (dashed arrow) is short 
Fig. 4 Postmortem neuropathological analysis of a tubulonodular lipoma (case 1 in Table 1). a Prenatal brain MRI performed at 36 weeks' gestation is T1-weighted coronal slice. The lipoma (arrow) is hyperintense. b Coronal macroscopic slice at the same level as (a). The interhemispheric fissure is completely filled with a yellowish tissue (arrows). The corpus callosum is not visible. $\mathbf{c}, \mathbf{d}$ Histological analysis of the lipoma. Hematoxylin (b) and S-100 (c) staining, 40-time magnification. The lipoma is composed of multivacuolated $(*)$ and univacuolated $\left(^{\circ}\right.$ ) adipose cells of various size and shape, corresponding to brown adipose tissue and white adipose tissue, respectively
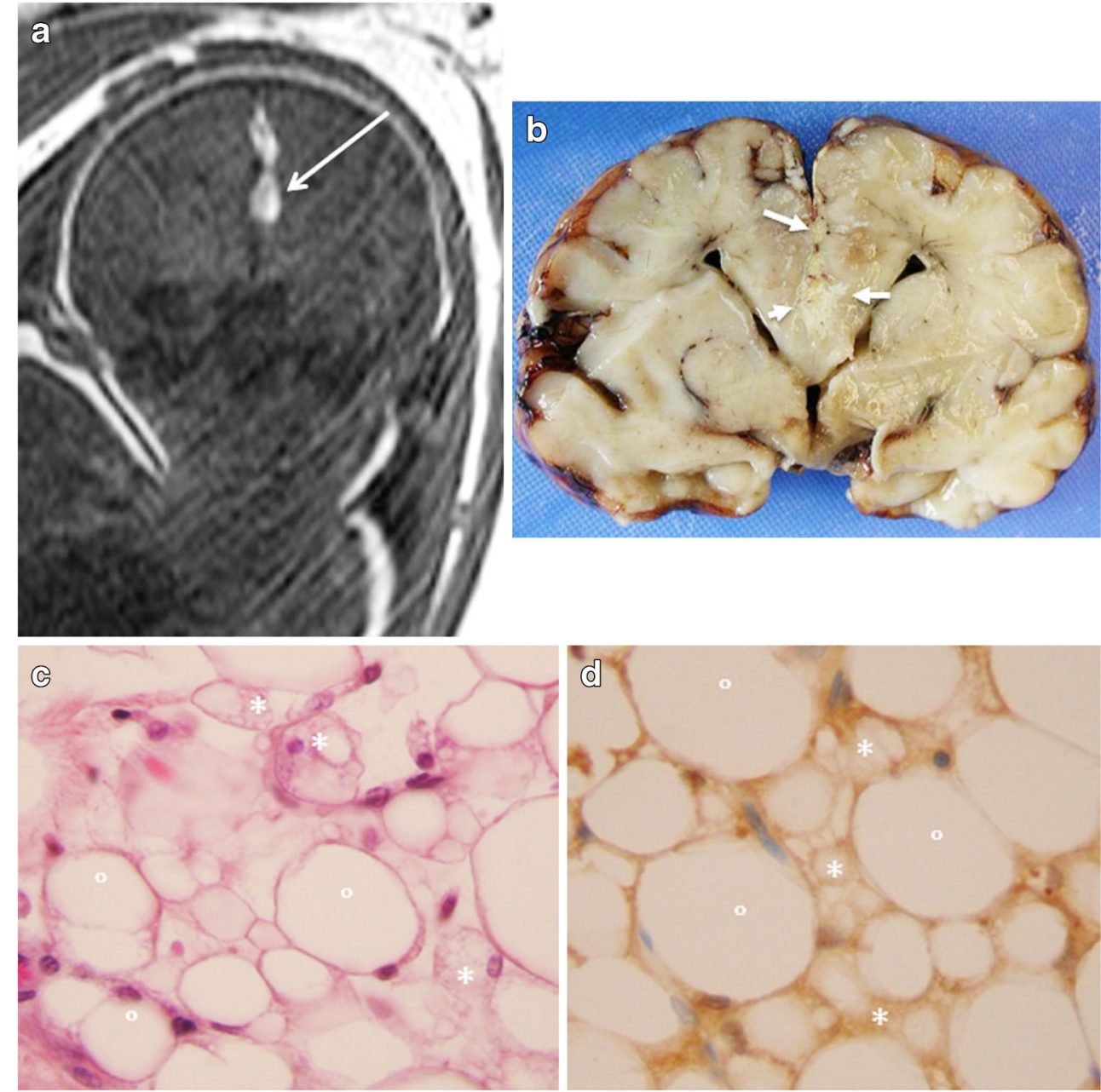

be anterior or posterior in location and the curvilinear type, which is thin, long and often posterior. The tubulonodular type is more common [1-11].

Pericallosal lipomas are often associated with corpus callosum dysgenesis, including complete and short corpus callosum and corpus callosum partial and complete agenesis [1-11]. Tubulonodular lipomas are more likely associated with other central nervous system impairments such as complete corpus callosum agenesis, encephaloceles, frontal bone defects and facial dysplasia. Conversely, curvilinear lipomas tend to be frequently asymptomatic and have a lower incidence of associated anomalies, with a corpus callosum being normal, sometimes incomplete or hypoplastic [10]. According to Tart and Quisling [10], both types of lipoma develop at different times during gestation with tubulonodular types developing earlier, which can account for this type being associated with more serious defects. Moreover, there is a higher risk of late-onset epilepsy when a tubulonodular lipoma is associated with polymicrogyria [9].

In our series, tubulonodular lipomas were more often associated with complete corpus callosum agenesis. Frontal hypoplasia and polymicrogyria were found in association with one case of tubulonodular lipoma (case 1). These findings are in accordance with the literature, interhemispheric lipomas having been reported to be associated with cortical anomalies such as cortical dysplasia, polymicrogyria or pachygyria-like lesions [16]. In our series, all patients had a normal neurological evaluation at birth and during the follow-up, except for one case of tubulonodular lipoma with complete agenesis of the corpus callosum. These findings agree with the literature. Therefore, the prenatal characterization of the pattern of the lipoma is an important prognostic marker, with curvilinear lipomas carrying a better outcome than tubulonodular lipomas.

In our series, interhemispheric hyperechogenicity was often the first anomaly detected by US and suggestive of a lipoma. This hyperechogenicity, particularly in the curvilinear type, may be rather thin and difficult to identify with certainty [8]. T1 hyperintensity was absent prenatally in our series in six fetuses with a curvilinear lipoma and appeared on postnatal MRI only. Moreover, in two additional fetuses with a tubulonodular lipoma, T1 hyperintensity was observed at prenatal MRI but markedly increased in size after birth. Atallah et al. [8] have recently reported four cases of curvilinear 
lipomas without prenatal $\mathrm{T} 1$ hyperintensity. In their study reporting seven cases of fetal pericallosal lipomas (including six tubulonodular lipomas), Ickowicz et al. [6] observed a postnatal growth of the lipoma in two patients. The possible involvement of other growth factors associated with the tumor or genetically determined was suggested [6]. Increase in the size of spinal lipomas has also been reported postnatally and may parallel the postnatal fatty growth of children [17].

Slice thickness is a factor that can be discussed to explain the absence of detection of the thinnest lipomas, which were all curvilinear. Atallah et al. [8] mentioned the poor spatial resolution in curvilinear lipomas without $\mathrm{T} 1$ hyperintensity. Nevertheless, in our study, two orthogonal planes were used in almost all cases to maximize the possibility of identifying pericallosal hyperintensity. Moreover, three out of the six curvilinear lipomas without prenatal T1 intensity had a lateral diameter between 3 and $6 \mathrm{~mm}$. This diameter was measured postnatally as prenatal measurement was not considered accurate enough in this type of lipoma. We can assume that this diameter might even be smaller prenatally. The slice thickness used on T1-weighted sequences was $4 \mathrm{~mm}$. Therefore, in these three cases, the slice thickness might account for the T1 hyperintensity of lipomas being missed prenatally. Conversely, in the other three cases with larger lateral diameter of the lipoma, the slice thickness and non-hyperintensity cannot be due to the lesion being very thin.

Another factor possibly accounting for the absence of prenatal $\mathrm{T} 1$ hyperintensity is the fat change related to a fat maturation process within lipomas. Indeed, two types of adipose tissue are known: Brown adipose tissue is the first fat tissue to appear during fetal development, at around mid-gestation. Its amount increases gradually until birth. Then, it progressively disappears and is replaced by white adipose tissue. Adult fat tissues are mainly composed of white adipose tissue while small foci of brown adipose tissue can remain in the supraclavicular regions. Brown adipose tissue is responsible for non-shivering thermogenesis, allowing heat production and adaptation to the cold temperatures. Conversely, white adipose tissue's role is energy storage [18-21].

In another study [22], we reported changes in $\mathrm{T} 1$ intensity of the subcutaneous fat tissue throughout gestation. A gradual increase of the $\mathrm{T} 1$ intensity of subcutaneous fat tissue was noticed with a threshold being observed at around 2931 weeks of gestation, according to the anatomical areas. Moreover, in the same study, analysis of pathological samples of subcutaneous fat tissue in 16 fetuses showed a predominance of brown adipose tissue before 29 weeks of gestation and then an inversion of the ratio between white and brown adipose cells. We assumed that the changes in the $\mathrm{T} 1$ intensity of subcutaneous fat tissue during pregnancy could be considered the consequence of this histological maturation from brown to white fat, with brown adipose tissue displaying $\mathrm{T} 1$ hypointensity and white adipose tissue displaying $\mathrm{T} 1$ hyperintensity. Similarly, in the current series, the subcutaneous fat layer displayed $\mathrm{T} 1$ hyperintensity in all fetuses, with a lower intensity in the cases with MRI being performed earlier in pregnancy, at 29 weeks of gestation. In our single case with available neuropathological examination at 36 weeks of gestation, a combination of white adipose tissue and brown adipose tissue with a predominance of the former was found. The process described in the subcutaneous fat tissue could be observed in lipomas as well. Histological analysis of pericallosal lipomas in fetuses is rarely available as the great majority of pregnancies with this malformation are not terminated, due to the good prognosis of this malformation.

\section{Conclusion}

Curvilinear types of lipomas are more often associated with a delayed $\mathrm{T} 1$ hyperintensity whose appearance seems to result from the size of the lipoma and from fetal fat maturation, with white adipose tissue, a mature tissue with high $\mathrm{T} 1$ intensity, being replaced by white adipose tissue, a mature tissue with high $\mathrm{T} 1$ intensity. This process seems to be faster in the tubulonodular type of lipoma.

Our study shows that if hyperechogenicity is associated with corpus callosum dysgenesis at prenatal US, the diagnosis of lipoma should be strongly suspected even if $\mathrm{T} 1$ hyperintensity is lacking. This has prognostic implications mainly in the curvilinear type of lipoma, due to outcome being better in corpus callosum dysgenesis associated with pericallosal lipomas than in isolated corpus callosum dysgenesis.

\section{Compliance with ethical standards}

Conflicts of interest None

\section{References}

1. Truwit CL, Barkovich AJ (1990) Pathogenesis of intracranial lipoma: an MR study in 42 patients. AJR Am J Roentgenol 155:855864

2. Yildiz H, Hakyemez B, Koroglu M et al (2006) Intracranial lipomas: importance of localization. Neuroradiology 48:1-7

3. Jabot G, Stoquart-Elsankari S, Saliou G et al (2009) Intracranial lipomas: clinical appearances on neuroimaging and clinical significance. J Neurol 256:851-855

4. Dean B, Drayer BP, Beresini DC et al (1988) MR imaging of pericallosal lipoma. AJNR Am J Neuroradiol 9:929-931

5. Erro Aguirre ME, de Lapiscina EHM (2014) Lipoma: an overview. In: Hayat M (ed) Tumors of the central nervous system. Springer, Dordrecht, pp 223-229

6. Ickowitz V, Eurin D, Rypens F et al (2001) Prenatal diagnosis and postnatal follow-up of pericallosal lipoma: report of seven new cases. AJNR Am J Neuroradiol 22:767-772 
7. Garel C (2004) Pathology of the midline. In: Garel C (ed) MRI of the fetal brain: normal development and cerebral pathologies. Springer Verlag, Berlin, pp 131-150

8. Atallah A, Lacalm A, Massoud M et al (2017) Limitations and pitfalls in prenatal diagnosis of pericallosal curvilinear lipoma based on a specific imaging pattern. Ultrasound Obstet Gynecol. https://doi.org/10.1002/uog.17400

9. Garel C, Moutard ML (2014) Main congenital cerebral anomalies: how prenatal imaging aids counseling. Fetal Diagn Ther 35:229-239

10. Tart RP, Quisling RG (1991) Curvilinear and tubulonodular varieties of lipoma of the corpus callosum: an MR and CT study. J Comput Assist Tomogr 15:805-810

11. Yilmaz MB, Genc A, Egemen E et al (2016) Pericallosal lipomas: a series of 10 cases with clinical and radiological features. Turk Neurosurg 26:364-368

12. Cignini P, Padula F, Giorlandino M et al (2014) Reference charts for fetal corpus callosum length: a prospective cross-sectional study of 2950 fetuses. J Ultrasound Med 33:1065-1078

13. Garel C, Cont I, Alberti C et al (2011) Biometry of the corpus callosum in children: MR imaging reference data. AJNR Am J Neuroradiol 32:1436-1443

14. Tilea B, Alberti C, Adamsbaum C et al (2009) Cerebral biometry in fetal magnetic resonance imaging: new reference data. Ultrasound Obstet Gynecol 33:173-181
15. Atanassova P (2001) Immunohistochemical expression of S-100 protein in human embryonal fat cells. Cells Tissues Organs 169: $355-360$

16. Niwa T, de Vries LS, Manten GT et al (2016) Interhemispheric lipoma, callosal anomaly, and malformations of cortical development: a case series. Neuropediatrics 47:115-118

17. Pierre-Kahn A, Zerah M, Renier D et al (1997) Congenital lumbosacral lipomas. Childs Nerv Syst 13:298-334

18. Frontini A, Cinti S (2010) Distribution and development of brown adipocytes in the murine and human adipose organ. Cell Metab 11: 253-256

19. Klingenspor M, Fromme T (2012) Brown adipose tissue. In: Symonds ME (ed) Adipose tissue biology. Springer, New York, pp 39-69

20. Saely CH, Geiger K, Drexel H (2012) Brown versus white adipose tissue: a mini-review. Gerontology 58:15-23

21. Symonds ME, Pope M, Budge H (2015) The ontogeny of brown adipose tissue. Annu Rev Nutr 35:295-320

22. Blondiaux E, Chougar L, Gelot A et al (2017) Normal MRI developmental patterns of fetal fat intensity. Pediatr Radiol. https://doi. org/10.1007/s00247-017-4038-Z 